\title{
Tendencias tecnológicas de depuración de lixiviados en rellenos sanitarios iberoamericanos ${ }^{*}$
}

\author{
Carlos Zafra-Mejía* \\ Diego Romero-Torres ${ }^{* *}$
}

\author{
Recibido: 25/04/2018 - Aceptado: 20/06/2019 \\ https://doi.org/10.22395/rium.v18n35a8
}

\begin{abstract}
Resumen
Este artículo identifica y analiza las tendencias en los enfoques y tecnologías de depuración del lixiviado utilizadas a nivel mundial e implementadas en rellenos sanitarios Iberoamericanos. Se utilizó una metodología de búsqueda sistemática de literatura entre los años 1990-2016. Se empleó también un índice de frecuencia de citación bibliográfica con el fin de establecer un orden de importancia promedio mediante cuartiles $(\mathrm{Q})$ para los enfoques y tecnologías detectadas en las bases de datos consultadas. Los resultados permiten identificar seis enfoques principales de depuración en Iberoamérica: biológico $(49,5 \%)>$ térmico $(17,0 \%)>$ natural $(9,80 \%)>$ fisicoquímico $(9,60 \%)>$ membranas $(8,90 \%)>$ recirculación $(5,20 \%)$. Se observa también una mayor frecuencia de citación del enfoque biológico a nivel Iberoamericano (49,5\%) que a nivel mundial $(34,1 \%)$. Los hallazgos sugieren el siguiente orden de importancia para las principales tecnologías de depuración en Iberoamérica: combinación con aguas residuales $(\mathrm{Q} 3)>$ lodos activados (Q3) > aireación (Q4) > humedales artificiales $(\mathrm{Q} 4)>$ recirculación $(\mathrm{Q} 4)$. En promedio, la tecnología de lodos activados exhibe probablemente una menor variación y mayores porcentajes de remoción de $\mathrm{DBO}_{5}$, DQO y N-NH $4\left(\mathrm{DBO}_{5}\right.$ : 54-98 \%; DQO: 44-90 \%; $\mathrm{N}-\mathrm{NH}_{4}: 81-99 \%$ ) en relación con la tecnología de combinación con aguas residuales $\left(\mathrm{DBO}_{5}:<5,0-93 \%\right.$; DQO: $<5,0-86 \%$; $\mathrm{N}_{\mathrm{NH}}:<5,0-34 \%$ ). El análisis geográfico sugiere que en la zona norte iberoamericana se reportan con mayor frecuencia las tecnologías de recirculación (13,5\%) y lodos activados (10,8 \%), mientras que en la zona sur se reportan más las tecnologías de humedales artificiales $(24,3 \%)$ y combinación con aguas residuales (16,2\%). En la última década de estudio (2006-2016) se reportaron con mayor frecuencia a nivel iberoamericano las tecnologías de depuración mediante humedales artificiales y lodos activados $(56,7 \%)$.
\end{abstract}

Palabras clave: Iberoamérica; lixiviado; relleno sanitario; tecnologías de depuración.

\footnotetext{
* Artículo de revisión escrito en el marco del proyecto de investigación Gestión ambiental del sistema de tratamiento de lixiviados producto de la biodegradación de residuos sólidos urbanos en un relleno sanitario colombiano desarrollado entre 2015 y 2017, con apoyo de los grupos de investigación para el Desarrollo Sostenible (Indesos) y de Ingeniería Ambiental (Giiaud). Entidades participantes: Universidad Distrital Francisco José de Caldas, Colombia.

** Ingeniero civil, magíster en Ingeniería Sanitaria y Ambiental, doctor en Ingeniería Ambiental. Profesor titular, Universidad Distrital Francisco José de Caldas, Bogotá, Colombia. Correo electrónico: czafra@udistrital.edu. co. Orcid: https://orcid.org/0000-0002-4061-4897

*** Administrador ambiental, magíster en Desarrollo Sustentable y Gestión ambiental. Profesor asistente, Universidad Distrital Francisco José de Caldas, Bogotá, Colombia. Correo electrónico: dcromerot@udistrital.edu.co. Orcid: https://orcid.org/0000-0003-4583-8758
} 


\title{
Technology Trends of Leachate Treatment in Ibero-American Landfills
}

\begin{abstract}
This paper identifies and analyses trends in approaches and technologies of leachate treatment used worldwide and implemented in Ibero-American landfills. A methodology of systematic literature research between 1990-2016 was used. A bibliographic citation frequency index was also used to establish an average order of importance using quartiles $(\mathrm{Q})$ for the approaches and technologies detected in the databases consulted. The results allowed to identify six main treatment approaches in Ibero-America: Biological (49.5\%) > thermal $(17.0 \%)>\operatorname{natural}(9.80 \%)>$ physicochemical $(9.60 \%)>$ membranes $(8.90 \%)>$ recirculation $(5.20 \%)$. There is also a higher citation frequency of the biological approach in Ibero-America (49.5\%) compared to its worldwide citation frequency $(34.1 \%)$. The findings suggest the following order of importance for the main treatment technologies in Ibero-America: Combination with wastewater (Q3) > activated sludge (Q3) $>$ aeration $(\mathrm{Q} 4)>$ artificial wetlands $(\mathrm{Q} 4)>$ recirculation $(\mathrm{Q} 4)$. On average, activated sludge technology possibly exhibits lower variation and higher removal rates of $\mathrm{BOD}_{5}, \mathrm{COD}$, and $\mathrm{N}-\mathrm{NH}_{4}\left(\mathrm{BOD}_{5}: 54-98 \%\right.$; COD: 44-90 \%; N-NH$:$ : 81-99\%) in relation with the technology of combination with wastewater $\left(\mathrm{BOD}_{5}\right.$ : $<5.0-93 \%$; COD: $<5.0-86 \%$; $-\mathrm{NH}_{4}:<5.0-34 \%$ ). Geographical analysis suggests that the technologies of recirculation (13.5\%) and activated sludge (10.8\%) are most frequently reported in northern Ibero-America, while in southern Ibero-America the most frequently reported are technologies of artificial wetland (24.3\%) and combination with wastewater (16.2\%). In the last decade of study (2006-2016), treatment technologies using artificial wetlands and activated sludge were most often reported in Ibero-America (56.7 \%).
\end{abstract}

Keywords: Ibero-America; leached; landfill; treatment technologies.

\section{Tendências tecnológicas de depuração de lixiviados em aterros sanitários ibero-americanos}

\section{Resumo}

Este artigo identifica e analisa as tendências nas abordagens e tecnologias de depuração do lixiviado utilizadas mundialmente e implementadas em aterros sanitários ibero-americanos. Utilizou-se uma metodologia de busca sistemática de literatura entre os anos 1990-2016. Empregou-se também um índice de frequência de citação bibliográfica com o objetivo de estabelecer uma ordem de importância média a partir de quartis (Q) para as abordagens e tecnologias detectadas nas bases de dados consultadas. Os resultados permitem identificar seis abordagens principais de depuração na Ibero-América: biológica $(49,5 \%)>$ térmica $(17,0$ $\%)>\operatorname{natural}(9,80 \%)>$ físico-química $(9,60 \%)>$ membranas $(8,90 \%)>$ recirculação $(5,20 \%)$. Observa-se também uma maior frequência de citação da abordagem biológica a nível ibero-americano (49,5\%) do que mundialmente $(34,1 \%)$. As descobertas sugerem a seguinte ordem de importância para as principais tecnologias de depuração na Ibero-América: combinação com águas residuais (Q3) > lodos ativados $(\mathrm{Q} 3)>$ aeração $(\mathrm{Q} 4)>$ áreas úmidas artificiais $(\mathrm{Q} 4)>$ recirculação (Q4). Em média, a tecnologia de lodos ativados exibe provavelmente uma menor variação e maiores porcentagens de remoção de $\mathrm{DBO}_{5}$, DQO e $\mathrm{N}-\mathrm{NH}_{4}$ ( $\mathrm{DBO}_{5}$ : 54-98 \%; DQO: 44-90\%; N-NH

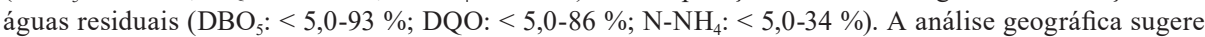
que na zona norte ibero-americana as tecnologias de recirculação $(13,5 \%)$ e lodos ativados $(10,8 \%)$ são reportadas com maior frequência, ao passo que na zona sul as tecnologias de áreas úmidas artificiais $(24,3 \%)$ e combinação com águas residuais (16,2 \%) são as mais reportadas. Na última década de estudo (2006-2016), as tecnologias de depuração por meio de áreas úmidas artificiais e lodos ativados (56,7 \%) foram mencionadas com maior frequência no contexto ibero-americano.

Palavras-chave: Ibero-América; lixiviado; aterro sanitário; tecnologias de depuração. 


\section{INTRODUCCIÓN}

Los impactos ambientales generados por el crecimiento y concentración de la población traen consigo un incremento en la producción de residuos sólidos urbanos (RSU), lo cual constituye una de las problemáticas ambientales más significativas a nivel mundial [1]. La gestión inadecuada de los RSU afecta directamente la salud de los habitantes en los puntos de generación, recolección, transporte y disposición final, y los recursos naturales allí existentes [2-3].

En la mayoría de los países iberoamericanos los rellenos sanitarios son el destino final más común de los RSU [4-5]. Uno de los problemas ambientales más significativos asociados con este tipo de instalaciones corresponde a los residuos líquidos generados durante su operación y clausura, los cuales se conocen técnicamente como lixiviados [6-7]. El lixiviado se ha convertido en uno de los principales desafíos de la ingeniería del tratamiento de residuos líquidos debido a sus elevadas cargas contaminantes (p. ej., materia orgánica y metales pesados), por ello destaca como una de las áreas de mayor interés en los rellenos sanitarios [7-9].

En la mayoría de los países latinoamericanos las tecnologías implementadas para el tratamiento de lixiviados no cumplen con las condiciones técnicas para controlar la contaminación ambiental (p. ej., en suelo, sistemas fluviales y agua subterránea) y las afectaciones para la salud pública [10-12]. Lo anterior incrementa los costos de operación de los sistemas de tratamiento y pone en riesgo el uso futuro de los recursos ambientales existentes [13-15]. Por lo tanto, resulta de especial interés estudiar las tecnologías de tratamiento de lixiviados desarrolladas a nivel mundial para identificar y evaluar los diferentes sistemas de depuración y visualizar tendencias locales para su implementación. En este contexto, España y Portugal son referentes para los restantes países iberoamericanos, debido a que poseen un elevado índice de desarrollo humano que los categoriza como países desarrollados (i. e., elevado nivel de vida, desarrollo industrial y comercial).

Diferentes investigaciones a nivel mundial han estudiado la depuración de los lixiviados en rellenos sanitarios. Existen investigaciones a escala de laboratorio $[9,16]$ como también investigaciones a escala real [3, 17-18], las cuales han obtenido resultados interesantes mediante diferentes tecnologías de depuración. Adicionalmente, se han combinado tecnologías para alcanzar mayores eficiencias de depuración [19]. Las tecnologías habitualmente reportadas por las investigaciones son las siguientes: (i) procesos biológicos (aerobio y anaerobio); (ii) sistemas naturales; (iii) recirculación de lixiviados; (iv) evaporación forzada; (v) tratamiento combinado con aguas residuales urbanas; (vi) stripping de amonio; (vii) sistemas de membrana (biorreactores-membranas y ósmosis inversa); (viii) procesos de oxidación avanzada (POA); (ix) ozonización; y (x) procesos Fenton y foto-Fenton [20-24]. 
Los estudios reportan que la eficiencia de remoción de contaminantes para las diferentes tecnologías de depuración del lixiviado depende de su composición química y de la evaluación de parámetros como la $\mathrm{DBO}, \mathrm{DQO}$, biodegradación (relación $\mathrm{DBO} /$ DQO), nitrógeno total, fósforo, hierro, mercurio, cromo, zinc y $\mathrm{pH}$. Igualmente, de su relación con las características y grado de estabilización de los RSU según la edad del relleno sanitario [25-29].

El objetivo principal de este artículo de revisión es identificar y analizar las tendencias en los enfoques y tecnologías de depuración del lixiviado utilizadas a nivel mundial e implementadas en rellenos sanitarios de RSU en Iberoamérica. Este estudio permite establecer un orden de importancia para las tecnologías de depuración en Iberoamérica según su frecuencia de citación en bases de datos científicas. Adicionalmente, analiza los principales parámetros físicos, químicos y biológicos considerados por cada tecnología de depuración del lixiviado (TDL). Finalmente, estudia la evolución geográfica y cronológica en el uso de cada TDL.

\section{MATERIALES Y MÉTODOS}

\subsection{Sistema de búsqueda bibliográfica}

Para el desarrollo de la revisión bibliográfica se utilizó una metodología de búsqueda sistemática acerca de enfoques y tecnologías de depuración de lixiviados en rellenos sanitarios de RSU. La investigación bibliográfica se desarrolló entre 1990 y el 2016. Se establecieron también dos escalas geográficas de investigación: mundial e iberoamericana. Las bases de datos utilizadas fueron (i) Google Académico, (ii) SienceDirect, y (iii) Scopus. Para la revisión bibliográfica se tuvieron en cuenta todos los campos de búsqueda de las bases de datos consultadas (i. e., título, resumen, palabras clave y todo el contenido del documento). Se consideraron documentos tales como artículos científicos, libros, memorias de congresos e informes de investigación.

\subsection{Sistema de análisis bibliográfico}

En el análisis bibliográfico se utilizó una metodología de clasificación mediante cuartiles [30], según fue la frecuencia de citación de los enfoques y tecnologías de depuración del lixiviado detectadas a nivel mundial e iberoamericano. De esta manera, se asumió que los enfoques y tecnologías de depuración más significativas fueron frecuentemente citadas o estudiadas. Esto también permitió estimar su importancia a través de su frecuencia de citación en documentos científicos. Aunque las anteriores hipótesis no fueron necesariamente ciertas, estas se emplearon en la presente investigación bibliográfica como lineamientos de búsqueda y análisis. 
A partir de lo anterior, se realizó la detección y análisis de las tecnologías de depuración de lixiviados reportadas a nivel mundial. Posteriormente, se realizó la detección y análisis de estas tecnologías a nivel iberoamericano y se estudiaron las variables más significativas para cada TD.

\subsubsection{Análisis a nivel mundial}

En la primera fase (fase 1) de detección y análisis a nivel mundial se utilizaron las siguientes combinaciones de palabras clave en inglés: (i) "treatment system" (sistema de tratamiento), (ii) "leachate" (lixiviado), y (iii) "landfill" (relleno sanitario). En esta fase se detectaron 27.319 documentos a nivel mundial. La base de datos con el mayor número de documentos detectados fue Google Académico (18.400 documentos; 67 $\%)$, seguida por ScienceDirect $(7.319 ; 27 \%$ ) y Scopus $(1.600 ; 6 \%)$.

Se desarrolló una segunda fase (fase 2) de detección y análisis de información a nivel mundial. En esta fase se revisaron los primeros 50 documentos reportados por la base de datos Scopus, para detectar mediante el filtro de búsqueda "Keyword" los enfoques y tecnologías de depuración más referenciados a nivel mundial. Durante esta fase se detectaron seis enfoques principales de depuración según su frecuencia de citación: (i) biológico (biological), (ii) fisicoquímico (physical-chemist), (iii) membranas (membranes), (iv) recirculación (recirculation), (v) térmico (termic), y (vi) natural (natural). Adicionalmente, se detectaron 20 tecnologías de depuración del lixiviado asociadas con los anteriores enfoques (tabla 1).

Posteriormente, se desarrolló una tercera fase (fase 3) de revisión bibliográfica en la cual se incluyeron palabras clave adicionales en relación con la fase 1. Las palabras clave adicionales fueron generadas a partir de las tecnologías de depuración detectadas a nivel mundial (tabla 1). En esta fase se utilizó un índice de frecuencia de citación [30] que relacionó el número de documentos detectados en las fases 1 y 3 . Esto se hizo con el fin de establecer un orden de importancia mediante cuartiles (Q) para las 20 tecnologías de depuración del lixiviado detectadas a nivel mundial. El índice tuvo una variación entre 0 y 1 . Por ejemplo, para la tecnología de lodos activados, en la base de datos Google Académico se detectaron 16.200 documentos en la fase 3 y 18.400 documentos durante la fase 1; de esta manera el índice de citación en esta base de datos fue 16.200/18.400 = 0,880 (Q1, primer cuartil de citación; ver tabla 2). El orden de importancia de las tecnologías de depuración se estableció a partir del valor promedio del cuartil obtenido en cada una de las bases de datos utilizadas. 
Tabla 1. Enfoques y tecnologías de depuración detectadas a nivel mundial

\begin{tabular}{|c|c|c|c|c|}
\hline \multirow{2}{*}{$N .^{\circ}$} & \multirow{2}{*}{ Enfoque tecnológico } & Porcentaje (\%) & \multirow{2}{*}{$N .^{\circ}$} & \multirow{2}{*}{ Tecnología de depuración } \\
\hline & & $(n=50)$ & & \\
\hline \multirow{5}{*}{1} & \multirow{5}{*}{ Biológico } & \multirow{5}{*}{34,1} & 1 & Lodos activados \\
\hline & & & 2 & Reactor UASB \\
\hline & & & 3 & Combinación con aguas residuales \\
\hline & & & 4 & Reactor ASBR \\
\hline & & & 5 & $\begin{array}{l}\text { Reactor de membrana } \\
\text { bioelectroquímica-BM }\end{array}$ \\
\hline \multirow{7}{*}{2} & \multirow{7}{*}{ Fisicoquímico } & \multirow{7}{*}{25,0} & 6 & Oxidación de grafeno magnético-MGO \\
\hline & & & 7 & $\begin{array}{l}\text { Oxidación con peróxido de hidrógeno y } \\
\text { ozono }\end{array}$ \\
\hline & & & 8 & Oxidación Fenton y Electrofenton \\
\hline & & & 9 & Oxidación catalítica de tribromofenol \\
\hline & & & 10 & Electrodiálisis reactor químico-UEC \\
\hline & & & 11 & Oxidación con persulfato \\
\hline & & & 12 & Reactor bioelectroquímico \\
\hline \multirow{4}{*}{3} & \multirow{4}{*}{ Membranas } & \multirow{4}{*}{15,9} & 13 & Ósmosis inversa \\
\hline & & & 14 & Nanofiltración \\
\hline & & & 15 & Ultrafiltración \\
\hline & & & 16 & Microfiltración \\
\hline 4 & Recirculación & 11,4 & 17 & Recirculación \\
\hline \multirow{2}{*}{5} & \multirow{2}{*}{ Térmico } & \multirow{2}{*}{6,80} & 18 & Evaporación forzada \\
\hline & & & 19 & Aireación \\
\hline 6 & Natural & 6,80 & 20 & Humedales artificiales \\
\hline Total & & 100 & & \\
\hline
\end{tabular}

Fuente: elaboración propia 
Tabla 2. Orden de importancia para las tecnologías de depuración a nivel mundial

\begin{tabular}{|c|c|c|c|c|c|c|c|c|c|c|}
\hline \multirow{3}{*}{ 㝕 } & \multirow{3}{*}{ Palabras clave } & \multicolumn{6}{|c|}{ Base de datos utilizada } & \multirow{3}{*}{$\begin{array}{c}\text { Índice } \\
\text { prome- } \\
\text { dio }\end{array}$} & \multirow{3}{*}{$\begin{array}{c}\text { Cuartil } \\
\text { prome- } \\
\text { dio }\end{array}$} & \multirow{3}{*}{$\begin{array}{l}\text { Cuartil- } \\
\text { Variación }\end{array}$} \\
\hline & & \multicolumn{2}{|c|}{$\begin{array}{c}\text { Google } \\
\text { Académico }\end{array}$} & \multicolumn{2}{|c|}{ Science Direct } & \multicolumn{2}{|c|}{ Scopus } & & & \\
\hline & & 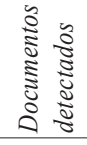 & 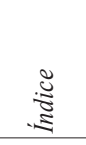 & 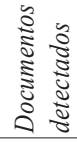 & 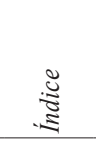 & 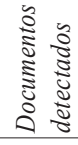 & 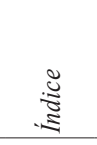 & & & \\
\hline 1 & $\begin{array}{l}\text { Lixiviado, sistemas de tra- } \\
\text { tamiento y relleno sanitario }\end{array}$ & 18.400 & - & 7.319 & - & 1.600 & - & - & - & - \\
\hline 3 & Lodos activados & 16.200 & $0,880^{\mathrm{a}}$ & 2.486 & 0,340 & 317 & 0,198 & 0,473 & Q3 & Q1-Q3-Q4 \\
\hline & $\begin{array}{l}\text { Combinación con aguas } \\
\text { residuales }\end{array}$ & 16.200 & 0,880 & 3.212 & 0,439 & 19 & 0,012 & 0,444 & Q3 & Q1-Q3-Q4 \\
\hline & Aireación & 15.700 & 0,853 & 1.824 & 0,249 & 119 & 0,074 & 0,392 & Q3 & Q1-Q4-Q4 \\
\hline & Reactor UASB & 16.500 & 0,897 & 493 & 0,067 & 76 & 0,048 & 0,337 & Q3 & Q1-Q4-Q4 \\
\hline & Evaporación forzada & 12.300 & 0,668 & 1.566 & 0,214 & 29 & 0,018 & 0,300 & Q3 & Q2-Q4-Q4 \\
\hline & Recirculación & 8.940 & 0,486 & 1.242 & 0,170 & 160 & 0,100 & 0,252 & Q3 & Q3-Q4-Q4 \\
\hline & Humedales artificiales & 9.130 & 0,496 & 1.005 & 0,137 & 105 & 0,066 & 0,233 & Q4 & Q3-Q4-Q4 \\
\hline & $\begin{array}{l}\text { Oxidación con peróxido de } \\
\text { hidrogeno y ozono }\end{array}$ & 8.930 & 0,485 & 520 & 0,071 & 40 & 0,025 & 0,194 & Q4 & Q3-Q4-Q4 \\
\hline & Ósmosis inversa & 5.810 & 0,316 & 1.003 & 0,137 & 62 & 0,039 & 0,164 & Q4 & Q3-Q4-Q4 \\
\hline & $\begin{array}{l}\text { Electrodiálisis reactor } \\
\text { químico UEC }\end{array}$ & 8.040 & 0,437 & 223 & 0,030 & - & $<0,001$ & 0,156 & Q4 & Q3-Q4-Q4 \\
\hline & $\begin{array}{l}\text { Oxidación Fentón y } \\
\text { electrofentón }\end{array}$ & 5.870 & 0,319 & 643 & 0,088 & 69 & 0,043 & 0,150 & Q4 & Q3-Q4-Q4 \\
\hline & $\begin{array}{l}\text { Oxidación de grafeno } \\
\text { magnético-MGO }\end{array}$ & 4.850 & 0,264 & 456 & 0,062 & 2 & 0,001 & 0,109 & Q4 & Q3-Q4-Q4 \\
\hline & Ultrafiltración & 3.610 & 0,196 & 738 & 0,101 & 17 & 0,011 & 0,103 & Q4 & Q4-Q4-Q4 \\
\hline & Nanofiltración & 2.690 & 0,146 & 445 & 0,061 & 29 & 0,018 & 0,075 & Q4 & Q4-Q4-Q4 \\
\hline & $\begin{array}{l}\text { Reactor de membrana } \\
\text { bioelectroquímica-BM }\end{array}$ & 2.120 & 0,115 & 629 & 0,086 & - & $<0,001$ & 0,067 & Q4 & Q4-Q4-Q4 \\
\hline & Microfiltración & 2.370 & 0,129 & 424 & 0,058 & 16 & 0,010 & 0,066 & Q4 & Q4-Q4-Q4 \\
\hline & $\begin{array}{l}\text { Oxidación catalítica } \\
\text { tribromofenol }\end{array}$ & 59 & 0,003 & 879 & 0,120 & 1 & 0,001 & 0,041 & Q4 & Q4-Q4-Q4 \\
\hline & Oxidación con persulfato & 1.120 & 0,061 & 147 & 0,020 & - & $<0,001$ & 0,027 & Q4 & Q4-Q4-Q4 \\
\hline & Reactor ASBR & 468 & 0,025 & 65 & 0,009 & 9 & 0,006 & 0,013 & Q4 & Q4-Q4-Q4 \\
\hline & Reactor bioeléctrico & 226 & 0,012 & 3 & $<0,001$ & 4 & 0,003 & 0,005 & Q4 & Q4-Q4-Q4 \\
\hline
\end{tabular}

Notas: a Índice de citación $=16 \cdot 200 / 18 \cdot 400=0,880$. 


\subsubsection{Análisis a nivel iberoamericano}

Se desarrolló una cuarta fase (fase 4) de investigación bibliográfica centrada en Iberoamérica. En esta fase se utilizaron las mismas bases de datos y tecnologías de depuración de lixiviados detectadas a nivel mundial (tabla 1) y se incluyeron las palabras clave en inglés "Iberoamérica" (Ibero-America) y "Latinoamérica" (Latin-America). Es decir, se evaluó la frecuencia de citación (Q) de las tecnologías de depuración en Iberoamérica y Latinoamérica. En esta fase se seleccionaron documentos científicos a partir de los siguientes criterios de inclusión de información: (i) reporte de tecnologías de depuración en rellenos sanitarios de RSU; (ii) reporte de eficiencia de remoción de contaminantes; y (iii) reporte geográfico y cronológico de la utilización de las tecnologías de depuración.

A partir de lo anterior, se analizaron las tecnologías de depuración que asociaron el mayor índice de citación promedio (Q3 y Q4) con un índice promedio mayor a 0,200 . De esta manera, en el análisis de las tecnologías de depuración del lixiviado se seleccionaron 37 documentos científicos. Algunos documentos se repitieron debido a que estos incluyeron más de una TDL. Finalmente, para la construcción del manuscrito se emplearon en total 50 referencias bibliográficas.

\subsection{Análisis estadístico}

Las siguientes pruebas estadísticas se utilizaron para el análisis de datos durante la investigación bibliográfica. Se utilizó la prueba de Shapiro-Wilk para contrastar la no normalidad del conjunto de datos ( $\mathrm{p}$-valor $<0,001$ ). La prueba estadística no paramétrica de Wilcoxon se empleó para analizar diferencias entre las variables listadas en la tabla 5. Es decir, esta prueba estadística fue utilizada para determinar diferencias o similitudes en los porcentajes de remoción de contaminantes $\left(\mathrm{DBO}_{5}, \mathrm{DQO}, \mathrm{N}-\mathrm{NH}_{4}\right.$, metales pesados y $\mathrm{pH}$ del efluente) entre las principales tecnologías de depuración del lixiviado en Iberoamérica. Se utilizaron también estadísticos descriptivos (media, mediana, máximo y mínimo), porcentajes e incrementos para estudiar la información. Todas las pruebas estadísticas se calcularon con un nivel de confianza del $95 \%$. Los análisis estadísticos se realizaron con el programa informático IBM-SPSS V. 18.0.

\section{RESULTADOS Y DISCUSIÓN}

\subsection{Enfoques y tecnologías de depuración a nivel mundial}

La investigación bibliográfica mostró el siguiente orden de importancia para los principales enfoques de depuración del lixiviado identificados a nivel mundial (tabla 1): i) biológico (34,1 \%); ii) fisicoquímico (25,0 \%); iii) membranas (15,9\%); iv) recirculación 
$(11,4 \%)$; v) térmico (6,8 \%); y vi) natural (6,8 \%). De esta manera, se evidenció una tendencia mundial en el uso (mayor citación) de los enfoques de tipo biológico y fisicoquímico para la depuración del lixiviado en rellenos sanitarios. Diferentes estudios desarrollados en Europa, Asia y Estados Unidos reportaron una tendencia similar a nivel mundial en el uso de estos enfoques de depuración del lixiviado [12, 31-33]. Estas investigaciones también reportaron que la anterior tendencia probablemente estuvo asociada con la secuencia de depuración del lixiviado más utilizada a nivel mundial: enfoque fisicoquímico más enfoque biológico.

Por otro lado, los resultados mostraron el siguiente orden de importancia para las principales tecnologías de depuración del lixiviado identificadas a nivel mundial (tabla 2): i) lodos activados (índice promedio = 0,473; Q3); ii) combinación con aguas residuales (índice $=0,444$; Q3); iii) aireación (índice $=0,392$; Q3); iv) reactor UASB (índice = 0,337; Q3); y v) evaporación forzada (índice $=0,300$; Q3). Por lo tanto, se observó una preferencia mundial por el uso de las tecnologías de lodos activados y combinación con agua residual para la depuración del lixiviado. Schaider et al. [31], así como Moreira et al. [33], reportaron una tendencia similar a nivel mundial en el uso de estas dos tecnologías de depuración del lixiviado. Los investigadores asociaron esta preferencia tecnológica con las eficiencias de remoción alcanzadas por dichas tecnologías de depuración, las cuales estuvieron en promedio entre el 80 y $90 \%$ para $\mathrm{DBO}_{5}$.

A partir de lo anterior, se analizaron en conjunto los enfoques y tecnologías de depuración del lixiviado a nivel mundial. Los resultados mostraron, en orden de importancia, que dentro del enfoque biológico las tecnologías más reportadas fueron lodos activados (Q3), combinación con aguas residuales (Q3) y reactor UASB (Q3). En promedio a nivel mundial, las tecnologías de lodos activados y combinación con aguas residuales fueron citadas cada una un 36,0 \% más en relación con la tecnología de reactor UASB (tabla 2). Con respecto al enfoque fisicoquímico, los hallazgos permitieron observar que las tecnologías más reportadas fueron oxidación con peróxido de hidrógeno y ozono (Q4), electrodiálisis mediante reactor químico - UEC (Q4), y oxidación Fenton y Electrofenton (Q4). En promedio, la tecnología de oxidación con peróxido de hidrógeno y ozono fue citada un $26,8 \%$ más en relación con las tecnologías de electrodiálisis mediante reactor químico - UEC, y oxidación Fenton y Electrofenton.

\subsection{Enfoques y tecnologías de depuración a nivel Iberoamericano}

Los resultados a nivel iberoamericano mostraron el siguiente orden de importancia en la frecuencia de citación de los enfoques de depuración del lixiviado: (i) biológico (49,5\%), (ii) térmico (17,0\%), (iii) natural (9,8\%), (iv) fisicoquímico (9,6\%), (v) mem- 
branas $(8,9 \%)$, y (vi) recirculación $(5,2 \%)$. Es así como se evidenció una preferencia en Iberoamérica por el enfoque de tipo biológico para la depuración del lixiviado. A nivel mundial este enfoque de depuración también fue el más reportado (tabla 1). Sin embargo, a nivel iberoamericano se prefirieron los enfoques de tipo térmico y natural antes que los enfoques fisicoquímico y membranas, los cuales se reportaron con mayor frecuencia a nivel mundial. Méndez et al. [20], Suárez y Cardona [21], y Qi et al. [22], reportaron una tendencia similar a nivel iberoamericano con relación al uso frecuente del enfoque biológico para la depuración del lixiviado. Al respecto, se reportó que el enfoque biológico se prefirió debido a su efectividad para la depuración de lixiviados jóvenes que presentaron elevadas concentraciones de ácidos grasos volátiles y una relación $\mathrm{DBO}_{5} / \mathrm{DQO}$ mayor a 0,4 , lo cual sugirió una elevada biodegradabilidad de estos $[22,26]$.

Por otro lado, los hallazgos permitieron visualizar el siguiente orden de importancia para las tecnologías de depuración del lixiviado más utilizadas en Iberoamérica: i) combinación con aguas residuales (índice promedio $=0,397$; Q3), ii) lodos activados (índice $=0,302 ; \mathrm{Q} 3$ ), iii) aireación (índice $=0,243 ; \mathrm{Q} 4$ ), iv) humedales artificiales (índice $=0,164 ; \mathrm{Q} 4$ ), y v) recirculación (índice $=0,156$; Q4). Se observó una preferencia iberoamericana por el uso de las tecnologías de combinación con aguas residuales y lodos activados (tabla 3). A nivel mundial estas tecnologías de depuración también fueron las más reportadas. No obstante, a nivel iberoamericano se prefirieron las tecnologías de humedales artificiales y recirculación antes que las tecnologías de reactor UASB y evaporación forzada, las cuales fueron reportadas con más frecuencia a nivel mundial.

Similarmente, algunas investigaciones a nivel iberoamericano [34-36] reportaron el uso frecuente de la tecnología de combinación con aguas residuales para la depuración del lixiviado debido a su bajo costo de implementación. Esta tecnología consistió en evacuar el lixiviado por la red pública de alcantarillado o transportarlo hasta una depuradora de aguas residuales municipales con el fin de reducir su carga contaminante mediante disolución [26, 37-38].

En relación con la tecnología de lodos activados, se reportó que sus principales ventajas fueron la elevada eficiencia de remoción de $\mathrm{DBO}_{5}$ (hasta del $90 \%$ ) y facilidad de operación [22, 39-40]. Las principales desventajas fueron sus costos de operación y las elevadas concentraciones de compuestos orgánicos e inorgánicos aportados por el lixiviado, lo cual disminuyó la sedimentación del lodo y generó problemas operacionales en las depuradoras municipales debido a la precipitación de óxidos de hierro [22, 39-40].

A partir de lo anterior, se analizaron en conjunto los enfoques y tecnologías de depuración del lixiviado a nivel iberoamericano. Los resultados sugirieron que dentro 
Tabla 3. Orden de importancia para las tecnologías de depuración a nivel iberoamericano

\begin{tabular}{|c|c|c|c|c|c|c|c|c|c|c|}
\hline \multirow{3}{*}{ 苟 } & \multirow[b]{3}{*}{ Palabras clave } & \multicolumn{6}{|c|}{ Base de datos utilizada } & \multirow{3}{*}{$\begin{array}{l}\text { Indice } \\
\text { promedio }\end{array}$} & \multirow{3}{*}{$\begin{array}{c}\text { Cuartil } \\
\text { promedio }\end{array}$} & \multirow{3}{*}{$\begin{array}{l}\text { Cuartil- } \\
\text { Variación }\end{array}$} \\
\hline & & \multicolumn{2}{|c|}{ Google Académico } & \multicolumn{2}{|c|}{ Science Direct } & \multicolumn{2}{|c|}{ Scopus } & & & \\
\hline & & 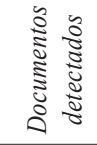 & 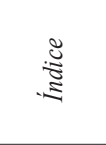 & 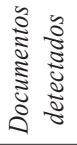 & 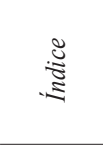 & 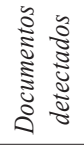 & 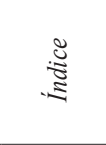 & & & \\
\hline 4 & $\begin{array}{l}\text { Lixiviado, sistemas de } \\
\text { tratamiento y relleno } \\
\text { sanitario }\end{array}$ & 13.169 & - & 179 & - & 66 & - & - & - & - \\
\hline 4 & $\begin{array}{l}\text { Combinación con agua } \\
\text { residual }\end{array}$ & 11.800 & $0,896 \mathrm{a}$ & 42 & 0,235 & 4 & 0,061 & 0,397 & Q3 & $\begin{array}{c}\text { Q1-Q4- } \\
\text { Q4 }\end{array}$ \\
\hline & Lodos activados & 6.320 & 0,480 & 49 & 0,274 & 10 & 0,152 & 0,302 & Q3 & $\begin{array}{c}\text { Q3-Q3- } \\
\text { Q4 }\end{array}$ \\
\hline & Aireación & 5.130 & 0,390 & 39 & 0,218 & 8 & 0,121 & 0,243 & Q4 & $\begin{array}{c}\text { Q3-Q4- } \\
\text { Q4 }\end{array}$ \\
\hline & Humedales artificiales & 3.850 & 0,292 & 17 & 0,095 & 7 & 0,106 & 0,164 & Q4 & $\begin{array}{c}\text { Q3-Q4- } \\
\text { Q3 }\end{array}$ \\
\hline & Recirculación & 2.040 & 0,155 & 26 & 0,145 & 11 & 0,167 & 0,156 & Q4 & $\begin{array}{c}\text { Q4-Q4- } \\
\text { Q3 }\end{array}$ \\
\hline & Evaporación forzada & 1.520 & 0,115 & 32 & 0,179 & - & $<0,001$ & 0,098 & Q4 & $\begin{array}{c}\text { Q4-Q4- } \\
\text { Q4 }\end{array}$ \\
\hline & $\begin{array}{l}\text { Oxidación con peróxido } \\
\text { de hidrogeno y ozono }\end{array}$ & 2.810 & 0,213 & 11 & 0,061 & - & $<0,001$ & 0,092 & Q4 & $\begin{array}{c}\text { Q4-Q4- } \\
\text { Q4 }\end{array}$ \\
\hline & Ósmosis inversa & 1.460 & 0,111 & 28 & 0,156 & - & $<0,001$ & 0,089 & Q4 & $\begin{array}{c}\text { Q4-Q4- } \\
\text { Q4 }\end{array}$ \\
\hline & Reactor UASB & 606 & 0,046 & 11 & 0,061 & 6 & 0,091 & 0,066 & Q4 & $\begin{array}{c}\text { Q4-Q4- } \\
\text { Q4 }\end{array}$ \\
\hline & $\begin{array}{l}\text { Oxidación Fentón y } \\
\text { electrofentón }\end{array}$ & 456 & 0,035 & 1 & 0,006 & 8 & 0,121 & 0,054 & Q4 & $\begin{array}{c}\text { Q4-Q4- } \\
\text { Q4 }\end{array}$ \\
\hline & Microfiltración & 601 & 0,046 & - & $<0,001$ & 7 & 0,106 & 0,051 & Q4 & $\begin{array}{c}\text { Q4-Q4- } \\
\text { Q4 }\end{array}$ \\
\hline & Nanofiltración & 598 & 0,045 & 3 & 0,017 & 6 & 0,091 & 0,051 & Q4 & $\begin{array}{c}\text { Q4-Q4- } \\
\text { Q4 }\end{array}$ \\
\hline & Ultrafiltración & 855 & 0,065 & - & 0,000 & 4 & 0,061 & 0,042 & Q4 & $\begin{array}{c}\text { Q4-Q4- } \\
\text { Q4 }\end{array}$ \\
\hline & $\begin{array}{l}\text { Reactor de membrana } \\
\text { biolectroquímica-BM }\end{array}$ & 704 & 0,053 & - & $<0,001$ & - & $<0,001$ & 0,018 & Q4 & $\begin{array}{c}\text { Q4-Q4- } \\
\text { Q4 }\end{array}$ \\
\hline & $\begin{array}{l}\text { Oxidación con } \\
\text { persulfato }\end{array}$ & 379 & 0,029 & 1 & 0,006 & 1 & 0,015 & 0,017 & Q4 & $\begin{array}{c}\text { Q4-Q4- } \\
\text { Q4 }\end{array}$ \\
\hline & $\begin{array}{l}\text { Electrodiálisis reactor } \\
\text { químico-UEC }\end{array}$ & - & $<0,001$ & 8 & 0,045 & - & $<0,001$ & 0,015 & Q4 & $\begin{array}{c}\text { Q4-Q4- } \\
\text { Q4 }\end{array}$ \\
\hline & Reactor ASBR & 68 & 0,005 & - & $<0,001$ & 1 & 0,015 & 0,007 & Q4 & $\begin{array}{c}\text { Q4-Q4- } \\
\text { Q4 }\end{array}$ \\
\hline & Reactor bioeléctrico & 84 & 0,006 & - & $<0,001$ & - & $<0,001$ & 0,002 & Q4 & $\begin{array}{c}\text { Q4-Q4- } \\
\text { Q4 }\end{array}$ \\
\hline & $\begin{array}{l}\text { Oxidación catalítica } \\
\text { tribromofenol }\end{array}$ & 35 & 0,003 & - & $<0,001$ & - & $<0,001$ & $<0,001$ & Q4 & $\begin{array}{c}\text { Q4-Q4- } \\
\text { Q4 }\end{array}$ \\
\hline & $\begin{array}{l}\text { Oxidación de grafeno } \\
\text { magnético-MGO }\end{array}$ & 5 & $<0,001$ & - & $<0,001$ & - & $<0,001$ & $<0,000$ & Q4 & $\begin{array}{c}\text { Q4-Q4- } \\
\text { Q4 }\end{array}$ \\
\hline
\end{tabular}

Nota: a Índice de citación $=11.800 / 13.169=0,896$.

Fuente: elaboración propia 
del enfoque biológico las tecnologías más utilizadas fueron las de combinación con aguas residuales (Q3), lodos activados (Q3) y reactor UASB (Q4). A nivel mundial se evidenció una tendencia similar (tabla 2).

En promedio, en el ámbito iberoamericano la TDL mediante combinación con aguas residuales fue citada $31,5 \%$ más en relación con la tecnología de lodos activados (tabla 3). Sin embargo, a nivel mundial la tendencia fue opuesta; la tecnología de lodos activados fue citada $6,53 \%$ más en relación con la tecnología de combinación con aguas residuales. Con respecto a los enfoques térmico y natural, los resultados mostraron las tecnologías de depuración mediante aireación (Q4) y humedales artificiales (Q4) como las más utilizadas a nivel iberoamericano, respectivamente. Lo anterior probablemente se debe al menor costo de implementación de este tipo de tecnologías de depuración del lixiviado, ya que por ejemplo a nivel latinoamericano, los presupuestos para instalaciones de saneamiento fueron más limitados [10, 12, 41].

Al realizar un análisis comparativo general entre las tendencias mundiales e iberoamericanas, los resultados mostraron un comportamiento similar en el uso del enfoque biológico como herramienta principal para la depuración del lixiviado. Al respecto, destacaron las tecnologías de lodos activados y combinación con aguas residuales, las cuales obtuvieron mayor frecuencia de citación. En el contexto mundial se dio preferencia por la tecnología de lodos activados, mientras que a nivel iberoamericano la preferencia fue por la tecnología de combinación con aguas residuales. Esta preferencia iberoamericana probablemente estuvo asociada con sus bajos costos de implementación, además de su simplicidad de operación. Es decir, probablemente se prefirió utilizar sistemas municipales de depuración ya construidos antes que invertir en nuevos sistemas independientes de depuración del lixiviado. Estudios internacionales reportaron una tendencia similar para reducir los costos de inversión mediante el uso de la tecnología de combinación con aguas residuales [42-43].

Sin embargo, se observaron diferencias para los demás enfoques detectados a nivel mundial e iberoamericano. Los resultados mostraron que el segundo enfoque de mayor importancia a nivel mundial para la depuración del lixiviado fue el fisicoquímico $(25,0 \%)$, mientras que en el ámbito iberoamericano fue el térmico (17,0\%). Lo mismo ocurrió para el tercer enfoque de depuración en los ámbitos mundial e iberoamericano. A nivel mundial se prefirió un enfoque de depuración mediante membranas $(15,9 \%)$, antes que un enfoque iberoamericano de tipo natural $(9,8 \%)$ mediante humedales artificiales. Nuevamente, los resultados sugirieron que estas preferencias iberoamericanas en los enfoques de depuración probablemente estuvieron asociadas con menores costos de construcción y operación de los sistemas de tratamiento. Por ejemplo, estudios internacionales reportaron que el enfoque de recirculación presentó menores costos 
de construcción y operación en relación con los enfoques de depuración fisicoquímico y membranas [26, 44-45].

\subsection{Análisis geográfico y cronológico en lberoamérica}

Se realizó un análisis geográfico iberoamericano para los principales enfoques y tecnologías de depuración del lixiviado según su frecuencia de citación. En este análisis geográfico se consideraron dos zonas continentales: norte (Centroamérica, España y Portugal) y sur (Suramérica). Los resultados mostraron que en la zona norte se dio el siguiente orden de importancia para los principales enfoques de depuración del lixiviado: biológico (13,5\%), recirculación (13,5\%), térmico $(5,41 \%)$, y natural $(5,41 \%)$. En la zona sur el orden de importancia fue el siguiente: biológico $(29,7 \%)$, natural $(24,3 \%)$, térmico $(5,41 \%)$, y recirculación (2,70 \%). De esta manera, los resultados evidenciaron un uso frecuente del enfoque biológico para la depuración del lixiviado en las dos zonas iberoamericanas de estudio (tabla 4).

Tabla 4: Distribución geográfica de tecnologías de depuración en Iberoamérica

\begin{tabular}{|c|c|c|c|c|c|}
\hline $\begin{array}{c}\text { Zona } \\
\text { geográfica }\end{array}$ & $N .^{\circ}$ & $\begin{array}{l}\text { Enfoque de } \\
\text { depuración }\end{array}$ & $\begin{array}{c}\text { Porcentaje } \\
(\%) \\
(n=37)\end{array}$ & Tecnología de depuración & $\begin{array}{c}\text { Porcentaje } \\
(\%) \\
(n=37)\end{array}$ \\
\hline \multirow{5}{*}{ Norte } & \multirow{2}{*}{1} & \multirow{2}{*}{ Biológico } & \multirow{2}{*}{13,5} & Lodos activados & 10,8 \\
\hline & & & & Combinación con aguas residuales & 2,70 \\
\hline & 2 & Recirculación & 13,5 & Recirculación & 13,5 \\
\hline & 3 & Térmico & 5,41 & Aireación & 4,41 \\
\hline & 4 & Natural & 5,41 & Humedales artificiales & 5,41 \\
\hline \multirow{5}{*}{ Sur } & \multirow{2}{*}{1} & \multirow{2}{*}{ Biológico } & \multirow{2}{*}{29,7} & Combinación con aguas residuales & 16,2 \\
\hline & & & & Lodos activados & 13,5 \\
\hline & 2 & Natural & 24,3 & Humedales artificiales & 24,3 \\
\hline & 3 & Térmico & 5,41 & Aireación & 5,41 \\
\hline & 4 & Recirculación & 2,70 & Recirculación & 2,70 \\
\hline
\end{tabular}

Notas: Zona geográfica norte: Centroamérica, España y Portugal;

Zona geográfica sur: Suramérica

Fuente: elaboración propia

Sin embargo, a nivel comparativo se observaron diferencias en la utilización de los demás enfoques de depuración del lixiviado en estudio. Por ejemplo, en la zona norte se prefirió el enfoque de recirculación (13,5\%) antes que el enfoque natural $(24,3 \%)$, el cual se reportó con mayor frecuencia en la zona sur. 
Con respecto a las tecnologías de depuración del lixiviado, los resultados mostraron el siguiente orden de importancia para la zona norte: recirculación $(13,5 \%)$, lodos activados $(10,8 \%)$, humedales artificiales (5,41\%), aireación (4,41\%), y combinación con aguas residuales $(2,70 \%)$. En la zona sur el orden de importancia fue el siguiente: humedales artificiales $(24,3 \%)$, combinación con aguas residuales $(16,2 \%)$, lodos activados (13,5\%), aireación $(5,41 \%)$ y recirculación $(2,70 \%)$.

Los resultados mostraron diferencias en el uso de las tecnologías de depuración entre las dos zonas de estudio. En la zona norte se reportaron con mayor frecuencia las tecnologías de recirculación y lodos activados, mientras que en la zona sur se reportaron más las tecnologías de humedales artificiales y combinación con aguas residuales. Los resultados insinuaron que estas diferencias probablemente se debieron a que en la zona norte se encuentran los dos países iberoamericanos más desarrollados (España y Portugal), los cuales tuvieron una mayor capacidad económica para implementar tecnologías de depuración más costosos (p. ej., lodos activados) en relación con la zona sur, en la cual se sitúan países en vías de desarrollo que implementaron tecnologías más económicas (p. ej., combinación con aguas residuales) [17, 32, 36, 46].

Adicionalmente, se realizó un análisis cronológico durante la última década del estudio (2006-2016) para evaluar la frecuencia de citación de cada una de las tecnologías de depuración del lixiviado en Iberoamérica. Los resultados mostraron el siguiente orden de importancia: humedales artificiales (29,7\%), lodos activados $(27,0 \%)$, combinación con agua residual (18,9\%), recirculación $(16,2 \%)$ y aireación $(8,11 \%)$. Por lo tanto, los resultados sugieren que durante la última década de estudio se referenciaron con mayor frecuencia las tecnologías de humedales artificiales y lodos activados, las cuales tuvieron un enfoque de depuración de tipo natural y biológico, respectivamente. Estas tecnologías de depuración del lixiviado representaron el $56,7 \%$ de los estudios en relación con las demás tecnologías iberoamericanas identificadas en la presente investigación (combinación con agua residual, recirculación y aireación).

\subsection{Eficiencia de las tecnologías en Iberoamérica}

\subsubsection{Enfoque biológico}

Los resultados a nivel iberoamericano mostraron que los sistemas de depuración del lixiviado mediante el enfoque biológico alcanzaron eficiencias de remoción de $\mathrm{DBO}_{5}$ entre $<5,0 \%$ y 98,0\%. Las tecnologías de depuración consideradas en el anterior análisis fueron aquellas de mayor frecuencia de citación (i. e., combinación con aguas residuales y lodos activados). De esta manera, se observó una amplia fluctuación en el porcentaje de remoción de $\mathrm{DBO}_{5}$ para el enfoque de depuración biológico (tabla 5). 
Tabla 5. Eficiencia de remoción de contaminantes en sistemas iberoamericanos de depuración del lixiviado $(n=37)$

\begin{tabular}{|c|c|c|c|c|c|c|c|c|c|c|c|c|}
\hline \multirow{2}{*}{$\begin{array}{l}\text { Enfoque } \\
\text { tecnológico }\end{array}$} & \multirow{2}{*}{$\begin{array}{c}\text { Tecnología de } \\
\text { depuración }\end{array}$} & & \multirow{2}{*}{$\begin{array}{l}\mathrm{DBO}_{5} \\
\text { (\$) }\end{array}$} & \multirow{2}{*}{$\begin{array}{c}D Q O \\
(\$)\end{array}$} & \multirow{2}{*}{$\mathrm{N}-\mathrm{NH}_{4}$} & \multirow{2}{*}{$\begin{array}{l}N \\
(\$)\end{array}$} & \multirow{2}{*}{$P(\$)$} & \multirow{2}{*}{$\begin{array}{l}p H \\
(\$)\end{array}$} & \multicolumn{4}{|c|}{ Metales pesados (\$) } \\
\hline & & & & & & & & & $\mathrm{Pb}$ & $\mathrm{Cd}$ & $\mathrm{Cr}$ & $\mathrm{Hg}$ \\
\hline \multirow{8}{*}{ Biológico } & \multirow{4}{*}{$\begin{array}{c}\text { Combinación } \\
\text { con aguas } \\
\text { residuales }\end{array}$} & Media & 32 & 32 & 14 & - & 13 & 7,9 & $<5$ & $<5$ & - & - \\
\hline & & Mediana & 72 & 61 & 24 & - & 14 & 7,9 & $<5$ & $<5$ & - & - \\
\hline & & Máximo & 93 & 86 & 34 & - & 18 & 8,7 & $<5$ & $<5$ & - & - \\
\hline & & Mínimo & $<5$ & $<5$ & $<5$ & - & 8 & 7,4 & $<5$ & $<5$ & - & - \\
\hline & \multirow{4}{*}{$\begin{array}{c}\text { Lodos } \\
\text { activados }\end{array}$} & Media & 71 & 61 & 87 & 81 & 35 & 7,8 & $<5$ & - & $<5$ & - \\
\hline & & Mediana & 72 & 59 & 83 & 81 & 45 & 7,9 & $<5$ & - & $<5$ & - \\
\hline & & Máximo & 98 & 90 & 99 & 82 & 73 & 8,5 & $<5$ & - & $<5$ & - \\
\hline & & Mínimo & 54 & 44 & 81 & 80 & 17 & 7,2 & $<5$ & - & $<5$ & - \\
\hline \multirow{4}{*}{ Natural } & \multirow{4}{*}{$\begin{array}{l}\text { Humedales } \\
\text { artificiales }\end{array}$} & Media & 34 & 57 & 79 & 14 & 12 & 7,5 & 17 & $<5$ & $<5$ & 10 \\
\hline & & Mediana & 55 & 58 & 80 & 20 & 44 & 7,5 & 18 & $<5$ & $<5$ & 11 \\
\hline & & Máximo & 98 & 82 & 89 & 82 & 98 & 9,1 & 23 & $<5$ & $<5$ & 12 \\
\hline & & Mínimo & $<5$ & 40 & 70 & $<5$ & $<5$ & 5,3 & 12 & $<5$ & $<5$ & 9 \\
\hline \multirow{4}{*}{ Recirculación } & \multirow{4}{*}{ Recirculación } & Media & 70 & 28 & - & - & 19 & 7 & $<5$ & - & - & - \\
\hline & & Mediana & 74 & 23 & - & - & 20 & 7 & $<5$ & - & - & - \\
\hline & & Máximo & 98 & 68 & - & - & 20 & 8 & 41 & - & - & - \\
\hline & & Mínimo & 50 & 12 & - & - & 18 & 6 & $<5$ & - & - & - \\
\hline \multirow{4}{*}{ Térmico } & \multirow{4}{*}{ Aireación } & Media & 37 & 46 & - & - & - & 9,9 & - & - & - & - \\
\hline & & Mediana & 51 & 37 & - & - & - & 11 & - & - & - & - \\
\hline & & Máximo & 85 & 87 & - & - & - & 11,5 & - & - & - & - \\
\hline & & Mínimo & 16 & 31 & - & - & - & 7,7 & - & - & - & - \\
\hline
\end{tabular}

Fuente: elaboración propia

Al comparar las dos tecnologías asociadas con este enfoque, los resultados mostraron diferencias significativas en los porcentajes de remoción a partir de la aplicación de una prueba de Wilcoxon ( $\mathrm{p}$-valor $<0,001$; gl. $=16$ ). Al respecto, se observó que la tecnología de lodos activados presentó una menor variación en el porcentaje de remoción de $\mathrm{DBO}_{5}(54-98$ \%) en relación con la tecnología de combinación con aguas residuales $(<5,0-93 \%)$. En el presente estudio, los resultados probablemente sugirieron una mayor estabilidad de la tecnología de lodos activados durante la eliminación de $\mathrm{DBO}_{5}$ en sistemas iberoamericanos de depuración del lixiviado. Se reportó también 
que esta TDL posiblemente permitió alcanzar mayores porcentajes de remoción de $\mathrm{DBO}_{5}$ [46-47]. Usualmente los lixiviados presentaron valores elevados de $\mathrm{DBO}_{5}$, por lo que al aplicar este tipo de tecnología biológica se pudieron alcanzar porcentajes de remoción superiores al $90 \%$. No obstante, la $\mathrm{DBO}_{5}$ remanente pudo ser mayor a $1.000 \mathrm{mg} / 1$ [17].

Adicionalmente, los resultados evidenciaron una tendencia similar de estas dos tecnologías biológicas en relación con los siguientes parámetros: $\mathrm{DQO}$ y $\mathrm{N}-\mathrm{NH}_{4}$ (tabla 5). Al respecto, una prueba de Wilcoxon mostró la existencia de diferencias significativas en los porcentajes de remoción en Iberoamérica ( $p$-valor $<0,001$; gl.-DQO = 16; gl.-N-NH $\mathrm{NH}_{4}=14$ ). Los hallazgos sugirieron que la tecnología de lodos activados probablemente presentó mayor estabilidad y porcentajes de remoción a nivel

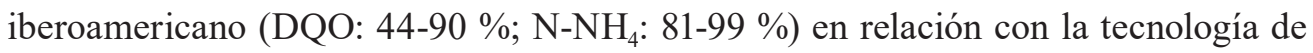
combinación con aguas residuales (DQO: $<5-86 \%$; $\mathrm{N}_{-} \mathrm{NH}_{4}:<5-34 \%$ ). Finalmente, los resultados no permitieron observar diferencias significativas en los porcentajes de remoción de metales pesados y $\mathrm{pH}$ del efluente para estas dos tecnologías biológicas de depuración ( $\mathrm{p}$-valor > 0,100; gl.-metales = 12; gl.-pH = 11).

\subsubsection{Enfoque natural}

Como se mencionó con anterioridad, la tecnología de humedales artificiales fue la más utilizada en Iberoamérica dentro del enfoque natural. Los resultados mostraron amplias fluctuaciones en los porcentajes de remoción para $\mathrm{DBO}_{5}(<5-98 \%)$, DQO (40-82\%), N (<5-82\%), y P (<5-98 \%). Estas fluctuaciones en los porcentajes de remoción probablemente estuvieron asociadas con las características del lixiviado a depurar. Es decir que en relación con la edad del lixiviado evaluado en los estudios seleccionados para la presente investigación bibliográfica (tabla 5), los resultados también mostraron de manera comparativa que la tecnología mediante humedales artificiales probablemente fue más eficiente para la remoción de metales pesados $(\mathrm{Pb}$ : 12-23 \%; Hg: 9-12\%; Cd y Cr: < $5 \%$ ) en relación con las tecnologías del enfoque biológico de combinación con aguas residuales y lodos activados ( $\mathrm{Pb}, \mathrm{Cd}$ y $\mathrm{Cr}:<5 \%)$. De esta manera, los resultados sugirieron emplear la tecnología mediante humedales artificiales como sistema de depuración terciario para la posible remoción de metales pesados en lixiviados de rellenos sanitarios. Algunos estudios [48-49] han reportado resultados similares para la tecnología de humedales artificiales en lixiviados de rellenos sanitarios de las ciudades de Bogotá (Colombia) y Quito (Ecuador), respectivamente.

A partir de lo anterior, esta TDL probablemente tuvo la ventaja de ser simple desde el punto de vista operativo, teniendo la posibilidad de lograr diferentes niveles de tratamiento, desde un pretratamiento hasta un tratamiento terciario [17]. Por 
ejemplo, la combinación de las tecnologías de lagunas de estabilización y humedales artificiales permitió resolver adecuadamente muchos de los problemas presentados con otras tecnologías, tales como la acumulación de precipitados, la formación de espumas, la toxicidad frente a los microorganismos, y las variaciones de las cargas hidráulicas y orgánicas [50].

\subsubsection{Enfoques de recirculación y térmicos}

Los resultados en Iberoamérica mostraron que la tecnología mediante recirculación logró eficiencias de remoción de $\mathrm{DBO}_{5}$, DQO, $\mathrm{P}$ y Pb entre 50 y $98 \%$, 12 y 68 \%, 18 y $20 \%$, y 5 y $41 \%$, respectivamente. Esta TDL -junto con la de lodos activadosfueron las que presentaron mayor estabilidad y porcentajes de remoción para $\mathrm{DBO}_{5}$ en Iberoamérica (tabla 5). Esta tecnología también reportó los mayores porcentajes de remoción para $\mathrm{Pb}(41 \%)$. Uno de los principales fines de la tecnología de recirculación fue controlar la libre dispersión de los lixiviados, estos se retornaron al relleno sanitario para su re-infiltración dentro de los RSU dispuestos. Por lo tanto, esta tecnología probablemente presentó ventajas debido al aumento del contenido de humedad en la masa de residuos, lo cual incrementó las tasas de degradación biológica y de recuperación de metano; otra de las ventajas reportadas en Iberoamérica fue su bajo costo de implementación [34].

Por otro lado, las tecnologías del enfoque térmico permitieron extraer el agua contenida en el lixiviado por medio de su evaporación. En el presente estudio, se identificó a nivel iberoamericano el uso de la tecnología de aireación para la depuración del lixiviado, la cual buscó evaporar el agua contenida en el lixiviado por calentamiento natural. Esta tecnología presentó ventajas económicas en su implementación, no obstante, presentó desventajas relacionadas con la eficiencia y remoción de los contaminantes [29]. Sin embargo, los resultados mostraron que esta tecnología presentó porcentajes de remoción de $\mathrm{DBO}_{5}$ y DQO entre 16 y $85 \%$, y 31 y $87 \%$, respectivamente. Finalmente, esta tecnología fue la que presentó las mayores fluctuaciones en el $\mathrm{pH}$ del efluente (entre 7,7 y 11,5) en relación con las demás tecnologías de estudio a nivel iberoamericano (tabla 5).

\section{CONCLUSIONES}

Los resultados a nivel mundial permiten identificar en orden de importancia seis enfoques principales de depuración del lixiviado según su frecuencia de citación (1990-2016): biológico $(34,1 \%)>$ fisicoquímico $(25,0 \%)>$ membranas $(15,9 \%)>$ recirculación $(11,4 \%)>$ térmico $(6,80 \%)>$ natural $(6,80 \%)$. Sin embargo, a nivel iberoamericano el orden de importancia de estos enfoques de depuración es el siguiente: 
biológico $(49,5 \%)>$ térmico $(17,0 \%)>$ natural $(9,80 \%)>$ fisicoquímico $(9,60 \%)>$ membranas $(8,90 \%)>$ recirculación $(5,20 \%)$. Es decir, se observa un mayor interés por o frecuencia de citación del enfoque de tipo biológico a nivel Iberoamericano $(49,5 \%)$ que a nivel mundial (34,1\%); existe también un mayor reporte de los enfoques de tipo térmico y natural a nivel iberoamericano en lugar de los enfoques de tipo fisicoquímico y membranas, los cuales son más citados a nivel mundial.

Adicionalmente, los hallazgos sugieren el siguiente orden de importancia para las tecnologías de depuración del lixiviado más reportadas a nivel iberoamericano: combinación con aguas residuales $(\mathrm{Q} 3)>$ lodos activados $(\mathrm{Q} 3)>$ aireación $(\mathrm{Q} 4)>$ humedales artificiales $(\mathrm{Q} 4)>$ recirculación $(\mathrm{Q} 4)$. Se observa entonces una mayor frecuencia de citación de tecnologías asociadas con el enfoque de depuración biológico: combinación con aguas residuales y lodos activados. A nivel mundial estas dos tecnologías de depuración también son las más reportadas. Sin embargo, a nivel iberoamericano se reportan con mayor frecuencia las tecnologías de humedales artificiales y recirculación en lugar de las tecnologías de reactor UASB y evaporación forzada, las cuales son más frecuentemente reportadas a nivel mundial.

El análisis geográfico en Iberoamérica sugiere que en la zona norte (Centroamérica, España y Portugal) se reportan con mayor frecuencia las tecnologías de recirculación $(13,5 \%)$ y lodos activados $(10,8 \%)$, mientras que en la zona sur (Suramérica) se reportan más las tecnologías de humedales artificiales $(24,3 \%)$ y combinación con aguas residuales $(16,2 \%)$. A nivel iberoamericano, en la última década de estudio (2006-2016) se reportaron con mayor frecuencia las tecnologías de depuración mediante humedales artificiales y lodos activados; estas tecnologías de depuración representan el $56,7 \%$ de los casos detectados.

En relación con la eficiencia de remoción de contaminantes en sistemas iberoamericanos, los resultados muestran que las principales tecnologías reportadas dentro del enfoque biológico (combinación con aguas residuales y lodos activados) presentan amplias fluctuaciones durante la remoción de $\mathrm{DBO}_{5}(<5-98,0 \%)$. Se observa también que la tecnología de lodos activados exhibe probablemente una menor variación o mayor estabilidad en el porcentaje de remoción de $\mathrm{DBO}_{5}(54-98 \%$ ) en relación con la tecnología de combinación con aguas residuales $(<5-93 \%)$. Similarmente, la tecnología de lodos activados presenta una mayor estabilidad y porcentajes de remoción para otros

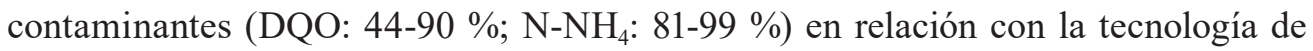

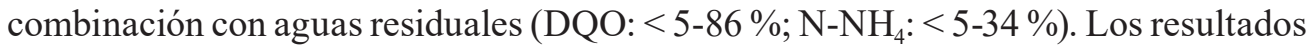
no permiten observar diferencias significativas en los porcentajes de remoción de metales pesados y $\mathrm{pH}$ del efluente para las principales tecnologías consideradas dentro del enfoque biológico. 
Finalmente, los resultados de la presente investigación permiten profundizar el conocimiento sobre las tendencias en la utilización de enfoques y tecnologías para la depuración de lixiviados en rellenos sanitarios de Iberoamérica, y son un punto de referencia para las instituciones ambientales encargadas de la gestión integral de los RSU, y para los diseñadores y operadores de sistemas de depuración de lixiviados.

\section{AGRADECIMIENTOS}

Los autores agradecen el apoyo académico a los grupos de investigación indesos y Giiaud de la Universidad Distrital Francisco José de Caldas (Colombia), así como los comentarios realizados por los árbitros debido a que permitieron mejorar el artículo significativamente.

\section{REFERENCIAS}

[1] V. N. Chaganti y D. M. Crohn. "Evaluating the relative contribution of physiochemical and biological factors in ameliorating a saline-sodic soil amended with composts and biochar and leached with reclaimed water," Geoderma, vol. 259, pp. 45-55, 2015.

[2] A. C. Patiño. "Gestión ambiental y tratamiento de residuos urbanos (manuscrito): propuesta para la zona metropolitana de Guadalajara a partir de las experiencias de la Unión Europea," Disertación Ph.D. Departamento de Geografía Humana, Universidad Complutense. Madrid, España, 2006.

[3] W. Lozano. "Uso del extracto de fique (Furcraea sp.) como coadyuvante de coagulación en tratamiento de lixiviados". Int. Contam. Amb., vol. 28, n. . 3, pp. 219-227, 2012.

[4] A. H. Bhatt, S. Altouqi, R. V. Karanjekar, M. S. Hossain, V. P. Chen y M. S. Sattler. "Preliminary regression models for estimating first-order rate constants for removal of BOD and COD from landfill leachate.” Environ. Tech. Innovation, vol. 5, pp. 188-198, 2016.

[5] M. D. Espinosa, M. López, A. Pellón, M. Robert, S. Díaz, A. González y A. Fernández. "Análisis del comportamiento de los lixiviados generados en un vertedero de residuos sólidos municipales de la ciudad de la Habana”, Int. Contam. Amb., vol. 26, n. . 4, pp. 313-325, 2010.

[6] K. Noguera y J. Olivero. “Los rellenos sanitarios en Latinoamérica: caso colombiano”. Acad. Colomb. Cienc. Exact. Fis. Nat., vol. 34, n. ${ }^{\circ}$ 132, pp. 347-356, 2010.

[7] M. S. Eljaiek, L. Torres y W. Bermúdez. "Alternativas de tratamiento de lixiviados aplicables al relleno sanitario parque ambiental Loma de los Cocos de la ciudad de Cartagena, Colombia". Hacia un sistema de gestión integral de los residuos sólidos, Cartagena: Redisa, pp. 1-6, 2013.

[8] G. K. Köfalusi y G. E. Aguilar, "Los productos y los impactos de la descomposición de residuos sólidos urbanos en los sitios de disposición final”, Gaceta ecológica, vol. 79, pp. 39-51, 2006. 
[9] C. M. Fernández, M. Ramírez, R. Rubio y A. Efraín, "Influencias de un relleno sanitario sobre la composición, abundancia y dispersión diurna de los peces en la Quebrada el Venado, corregimiento de Córdoba, Municipio de Buenaventura (Valle del Cauca, Colombia)", Acad. Colomb. Cienc. Exact. Fis. Nat., vol. 35, n. ${ }^{\circ}$ 135, pp. 213-224, 2011.

[10] D. E. Martínez y M. Osterrieth, "Hydrogeochemistry and pollution effects of an aquifer in Quaternary loess like sediments in the landfilling area of Mar del Plata, Argentina", Fac. Ing. Univ. Antioquia, n. ${ }^{\circ}$ 66, pp. 9-23, 2013.

[11] H. Zhong, Y. Tian, Q. Yang, M. L. Brusseau, L. Yang y G. Zeng, "Degradation of landfill leachate compounds by persulfate for groundwater remediation," Chem. Engin. Journal, vol. 307, pp. 399-407, 2016.

[12] Z. Ye, H. Zhang, L. Yang, L. Wu, Y. Qian, J. Geng y M. Chen. "Effect of a solar Fered-Fenton system using a recirculation reactor on biologically treated landfill leachate", J. Hazard Mat, vol. 319, pp. 51-60, 2016.

[13] A. Álvarez y J. H. Suárez. "Tratamiento biológico de lixiviados de rellenos sanitarios", Respuestas, vol. 11, n. ${ }^{\circ} 1$, pp. 24-32, 2006.

[14] J. A. Dávila y F. M. Granda, "Evaluación comparativa en una planta a escala piloto de lodos activados de aireación prolongada en el tratamiento de lixiviado de relleno sanitario municipal diluido con agua residual", Tesis de pregrado en Ingeniería Sanitaria, Facultad de Ingeniería Ambiental. Universidad Nacional de Ingeniería, Lima, Perú, 2013.

[15] L. Borzacconi, I. López, E. Arcia, L. Cardelin, A. Castagna y M. Viñas. "Comparación de tratamientos aerobios y anaerobios aplicados a lixiviado de relleno sanitario", en Consolidación para el desarrollo. XXV Congreso Interamericano de Ingeniería Sanitaria y Ambiental Aidis, México D.F., 1996, pp. 1-8.

[16] I. M. Noeggerath y M. A. Salinas Castillo, "Análisis comparativo de tecnologías para el tratamiento de lixiviados en rellenos sanitarios". Tesis de maestría, Universidad Veracruzana, Veracruz, México, 2011, pp. 1-9.

[17] A. G. Martínez, W. Padrón, O. Rodríguez, O. Chiquito, M. A. Escarola, J. M. Hernández y J. Martínez, "Alternativas actuales del manejo de lixiviados", Avan. Química, vol. 9, n. ${ }^{\circ}$ 1, pp. 37-47, 2014.

[18] A. Pellón, M. López, M. D. Espinosa y O. González, "Propuesta para tratamiento de lixiviados en un vertedero de residuos sólidos urbanos", Ingen. Hidra. Amb., vol. 36, n. ${ }^{\circ}$ 2, pp. 3-16, 2015.

[19] P. Mohammad y G. Weichgrebe, "Municipal landfill leachate characteristics and feasibility of retrofitting existing treatment systems with deammonification e - A full scale survey", Environ. Manag., vol. 187, pp. 354-364, 2017.

[20] R. I. Méndez, E. R. Castillo, M. R. Sauri, C. A. Quintal, G. Giácoman y B. Jiménez, “Comparación de cuatro tratamientos fisicoquímicos de lixiviados”, Int. Contam. Amb., vol. 25, n. 3 , pp. 133-145, 2009. 
[21] E. Suárez y S. A. Cardona, "Remoción de nitrógeno de lixiviados de un relleno sanitario mediante un sistema pasivo biológico secuencial", Dyna, vol. 80, n. ${ }^{\circ}$ 178, pp. 37-43, 2012.

[22] Z. Qi, T. Bao, Z. Xuan, G. Abbas, F. Cheng y H. Ruo. "Investigation on characteristics of leachate and concentrated leachate in three landfill leachate treatment plants". Waste Manag., vol. 33, n. ${ }^{\circ} 11$, pp. 2277-2286, 2013.

[23] P. Torres, L.E. Barba-Ho, C. Ojeda, J. Martínez y Y. Castaño, "Influencia de la edad de lixiviados sobre su composición físico-química y su potencial de toxicidad”, U.D.C.A Act. Div. Cient., vol. 17, n. ${ }^{\circ}$ 1, pp. 245-255, 2014.

[24] C. Ricordel y H. Djelal, "Treatment of landfill leachate with high proportion of refractory materials by electrocoagulation: System performances and sludge settling characteristics," Environ. Chem. Engin., vol. 2, n. . 3, pp. 1551-1557, 2014.

[25] J. D. Pérez, “Aplicación y evaluación de un reactor de contactores biológicos rotativos ( $\mathrm{RBC}$ o biodiscos) a escala laboratorio como tratamiento de los lixiviados generados en el relleno sanitario de La Pradera". Tesis de M.S., Facultad de Ingeniería, Ingeniería Urbana, Universidad de Medellín, Medellín, Colombia, 2010.

[26] E. Giraldo, "Tratamiento de lixiviados de rellenos sanitarios: avances recientes", Rev. Ing., vol. 14, pp. 44-55. 2001.

[27] M. Corena, "Sistemas de Tratamiento para Lixiviados Generados en Rellenos Sanitarios", Tesis de pregrado, Departamento de Ingeniería Civil, Facultad de Ingeniería, Universidad de Sucre, Sucre, Colombia, 2008.

[28] J. R. Laines y R. H. Adams, "Desarrollo y aplicación de un nuevo coadyuvante-coagulante orgánico en el proceso de coagulación-floculación del lixiviado de un relleno sanitario", Aidis Ing. Cien. Amb., vol. 1, n. ${ }^{\circ}$ 4, pp. 1-31, 2008.

[29] R. Méndez, A. Novelo, V. Coronado, E. Castillo y M. R. Sauri, "Remoción de materia orgánica y metales pesados de lixiviados por flotación con aire disuelto", Rev. Ing., vol. 12, n. 1 , pp. 13-19, 2008.

[30] C. Zafra, J. Temprano e I. Tejero, "The physical factors affecting heavy metals accumulated in the sediment deposited on road surfaces in dry weather: a review", Urb. Wat. Journal, vol. 14, n. ${ }^{\circ}$ 6, pp. 639-649, 2016.

[31] L. A. Schaider, J. M. Ackerman y R. A. Rudel, "Septic systems as sources of organic wastewater compounds in domestic drinking water wells in a shallow sand and gravel aquifer", Sci. Total Environ., vol. 547, pp. 470-481, 2016.

[32] T. F. Silva, P. A. Soares, D. R. Manenti, A. Fonseca, I. Saraiva, R .A. Boaventura y V. J. Vilar, "An innovative multistage treatment system for sanitary landfill leachate depuration: Studies at pilot-scale”, Sci. Total Environ., vol. 576, pp. 99-117, 2016.

[33] F. C. Moreira, J. Soler, A. Fonseca, I. Saraiva, R. A. Boaventura, E. Brillas y V. J. Vilar, "Incorporation of electrochemical advanced oxidation processes in a multistage treatment system for sanitary landfill leachate”, Wat. Res., vol. 81, pp. 375-387, 2015. 
[34] L. E. Barba, P. Torres, J. A. Rodríguez, L. F. Marmolejo y C. A. Pizarro, "Influencia de la incorporación de lixiviados sobre la biodegradabilidad anaerobia de aguas residuales domésticas", Rev. Ing. Inv., vol. 30, n. ${ }^{\circ}$ 1, pp. 75-79, 2010.

[35] A. M. Mendoza, "Planta de tratamiento de lixiviados-parque ambiental Los Pocitos", en II Simposio Iberoamericano de Ingeniería de Residuos Ingeniería. Red de Ingeniería en Saneamiento Ambiental. Barranquilla, pp. 55, 2009.

[36] A. Gálvez, "Aplicabilidad de procesos de coagulación-floculación y de sistema de biopelícula en el tratamiento de lixiviados de vertederos de residuos urbanos", Disertación de Ph.D., Departamento de Ciencias Ambientales, Licenciatura en Ciencia Ambiental, Universidad de Granada, Ganada, España, 2008.

[37] L. A. Fuentes y J. J. Palacio, "Evaluación del sistema alternativo de evaporación forzada de lixiviados para el relleno sanitario “Don Juanito" de Villavicencio, Meta”. Tesis de pregrado, Facultad de Ingeniería Sanitaria y Ambiental, Universidad de la Salle, Bogotá, Colombia, 2006.

[38] P. Torres, "Impacto de la incorporación de lixiviados en el arranque de reactores anaerobios al tratar aguas residuales domésticas", Rev. Ing. Univ., vol. 14, no. 2, pp. 313-326, 2010.

[39] T. F. Silva, A. Fonseca, I. Saraiva, V. J Vilar y R. A. Boaventura, "Mejora de la biodegradabilidad de un lixiviado después de lagunaje biológica usando una reacción solar impulsado foto-Fenton, y más en combinación con un proceso biológico de lodos activados, a escala pre-industrial". Inv. Agua, vol. 47, no. 10, pp. 3543-3557. 2013.

[40] T. F. Silva, P. A. Soares, D. R. Manenti, A. Fonseca, I. Saraiva, R. A. Boaventura, y V. J. Vilar. "An innovative multistage treatment system for sanitary landfill leachate depuration: Studies at pilot-scale". Sci. Total Environ, vol. 576, pp. 99-117, 2015.

[41] F. M. Ferraz, A. T. Bruni, J. Povinelli y E. M. Vieira. "Leachate/domestic wastewater aerobic co-treatment: A pilot-scale study using multivariate analysis”. J. Environ Manag., vol. 166, pp. 414-419, 2016.

[42] H. Sun, L. Ü. Xintao, Y. Peng, S. Wang y J. Ma. "Long-term nitritation performance of ammonium-rich landfill leachate". Chin. J. Chem. Eng., vol. 23, no. 11, pp. 1888-1893, 2015.

[43] K. M. Gros, H. Blum, H. Jernstedt, G. Renman, S. Rodríguez, P .Haglund L. y Ahrens, L. "Screening and prioritization of micropollutants in wastewaters from on-site sewage treatment facilities". J. Hazard Mat., vol. 328, pp. 37-45, 2016.

[44] R. Espinace, J. Palma y J. M. Sánchez. "Experiencias de aplicación de modelos para la determinación de los asentamientos de rellenos sanitarios". XI Congreso Panamericano de Mecánica de Suelos e Ingeniería de Fundaciones. Foz de Iguazu, Brasil. 199, pp. 8-12.

[45] G. I. González, E. Rustrián, E. Houbron y A. Zamora, "Impacto de la tasa de humedad en la biodegradación de los residuos sólidos urbanos de la ciudad de Veracruz, México”, Latin. Rec. Nat., vol. 4, n. ${ }^{\circ}$ 3, pp. 336-341, 2008. 
[46] C. V. Droppelmann y M. Oettinger, "Tratamiento en Lodo Activado del Lixiviado de un Relleno Sanitario”, Inf. Tecnol., vol. 20, n. ${ }^{\circ}$ 1, pp. 11-19, 2009.

[47] N. Valencia y N. Rivera. "Estudio preliminar para el tratamiento de lixiviados en un reactor de lodos activados”. Tesis de pregrado, Departamento de Ingeniería Química, Facultad de Ingeniería y Arquitectura, Universidad Nacional de Colombia, Manizales, Colombia, 2003.

[48] C. A. Madera, E. J. Peña y J. A. Solarte, "Effect of heavy metal concentration on the physiological responses and heavy metal accumulation of three tropical plant species used for phytoremediation of landfill leachate", Rev. Ing. Compet., vol. 16, n. ${ }^{\circ}$ 2, pp. 179-188, 2014.

[49] A. Guevara, L. Guanoluisa y E. de la Torre, "Diseño de Sistemas de Tratamiento de Lixiviados del Relleno Sanitario", Pol., vol. 34, n. ${ }^{\circ}$ 1, pp. 1-8, 2014.

[50] A. E. Cortés Sandoval, "Evaluación del desempeño de humedales construidos subsuperficial de flujo horizontal sembrados con especies nativas tropicales para la eliminación de $\mathrm{Cr}$ (VI) y Cd (II) de lixiviado de relleno sanitario", Disertación Ph.D. Esc. Ing. Rec. Nat. y Amb. Fac. Ing. Sanitaria y Amb. Univ. Valle., Cali, Colombia, 2014. 